\title{
Museums - What They Can and Should Be Doing
}

\author{
Charles H. Lindsey \\ Museum of Science and Industry, Manchester (volunteer) \\ chl@clerew.man.ac.uk
}

\begin{abstract}
Museums have a duty, not only to preserve the Objects in their care, but also to preserve the skills and the mindsets of those who created them; furthermore, those concepts must somehow be conveyed to the visiting public. I shall give examples of how this can be achieved when illustrating how the early computers paved the way for the ubiquitous devices that we know today. These will include interactive gadgets to show the underlying principles, and videos of the objects in use.
\end{abstract}

Keywords: Museums, purpose, history, mindsets.

\section{What Is the Purpose of a Museum?}

I suggest there are four main objectives:

1. To inform and educate the visiting Public as to the nature and significance of the Objects on display.

2. To enable serious researchers, perhaps far in the future, to examine the Objects in order to discover their precise nature, history, capabilities and manner of use.

No great disagreement about those two, I would imagine.

3. To preserve the skills necessary for the maintenance and operation of the Objects. That one is not so obvious. Some Museums do it well. For example, the skills of operating cotton machinery are well preserved at MOSI $^{1}$, Styal Mill $^{2}$ and, most effectively, at Helmshore ${ }^{3}$. When it comes to computing, little happens except when pressurized by the $\mathrm{CCS}^{4}$. MOSI has the reconstructed "Baby" (a replica of the world's first stored-program computer) which is preserving the art of working with thermionic valves - and just in time given that the people with the necessary skills are dying off at an unseemly rate.

Sometimes the pioneers have all died off, and then the skills have to be rediscovered by Trial and Error, as I am discovering myself with Hartree's Differential Analyser (where many times the Trial has indeed turned out to be an Error).

\footnotetext{
The Museum of Science and Industry, Manchester.

Quarry Bank Mill, Cheshire.

Helmshore Mills Textile Museum, Lancashire.

The Computer Conservation Society.
} 
TNMOC $^{5}$ is the shining example, expressly created for this purpose, but the Science Museum ${ }^{6}$ has an ambivalent approach. It has the working Pegasus (as a result of CCS pressure, but beset with Health and Safety issues). Many years ago, it obtained a large piece of Hartree's Differential Analyser restored to (allegedly) working order, but after around 12 months it lost interest and it has lain idle ever since. It constructed a replica of Babbage's (never built) $2^{\text {nd }}$ difference engine, and every day someone would come and crank the handle and show it in operation. Nowadays, that handle is only cranked when somebody especially requests it.

4. To preserve the "mindset" or "world-view" of the people who made the Objects.

And that one, I suspect, goes largely unrecognised, but it is the one I want to explore in this paper. It concerns what they thought they were trying to achieve. It concerns their view of the tools available for the purpose. It concerns the methods they used for going about their task - the problems they considered it important to address.

Now this "mindset" is what I want to convey to the Public. I do not expect the Public to be especially interested in the skills as such, though they may well appreciate seeing the results of exercising them. And note also that the "mindset" is a function of time, so it will have varied throughout History.

\section{Historical Perspectives}

\subsection{When Did History Start?}

Well the Romans had the abacus (a digital device) and they weighed things by sliding weights on a long lever (an analogue device).

But it was in the $19^{\text {th }}$ Century when things really got going, with Babbage's illfated projects, and with the need for astronomical tables as the driving force. At the start of the $20^{\text {th }}$ Century we had punched cards and mechanical calculators, such as the Brunsviga. Then came wheel-and-disc integrators, much used during the war for bomb sights and anti-aircraft control; and finally electronic devices and, above all, the Von Neumann model of the stored program computer.

\subsection{When Should History End?}

Initially, applications were purely arithmetical, whether for scientific or commercial purposes. Artificial intelligence was just a twinkle in the eye of Alan Turing and language translation in the eye of Andrew Booth; and that was it. Computers were large and bulky; you could look inside them and see how they were constructed, and they had lots of flashing lights and knobs to twiddle (a cartoonist's dream!) so you could see and control what was happening.

5 The National Museum of Computing, Bletchley Park.

6 The Science Museum, London. 
And then, around 1980, two fundamental changes occurred (not entirely unrelated). The first was the Integrated Circuit - a computer on a chip. Now you cannot see what is inside. IC design is an esoteric art practised by Intel, ARM and a few others. I cannot foresee how, in 200 years' time, anyone will have preserved, or even be able to rediscover, that art. ICs are just not museum-friendly - right at the end of the gallery you may see a computer chip opened up, with a large magnifying glass (or even a microscope) in front of it, and that is about as much as you can do.

The second change was the WIMP interface, coupled with the ability to manipulate images and sounds and to transmit them over the internet. Computers are now mostly about Communication, and Communications are mostly performed by Computers. Alan Turing has met Claude Shannon, and you can hardly tell the difference between them.

Museums are meant to record history, but things are now changing so rapidly that they cannot possibly keep up. So I therefore propose that we should regard Computer History, and the involvement of Museums with it, as having ended when these changes arrived. Someday, when the dust has settled and the perspectives have become clearer, that situation can be reviewed. But it won't be regarded as "Computer" History anymore, even though it is clear that whatever it is would never have come about if real computers hadn't happened first.

\section{Why We Need Working Machines}

There is a well-known saying: A Picture is worth a thousand Words. I would add two corollaries to this:

1. A Live Demonstration is worth a thousand Pictures

2. Hands-on experience is worth a thousand Live Demonstrations

Clearly, a working machine fulfils our aim to preserve those skills. But even working machines cannot be demonstrated continuously, so their interpretation is still important. We can distinguish several situations:

\subsection{Purely Static Objects}

Early manufacturers took great pride in enclosing their computers in shiny cabinets so you could not see what was inside. So at least the doors should be left open, or the covers left off, if there is something to be seen within. If it is a chain printer, then you need to be able to see the chain. If it is a hard disc, then it needs to be sectioned so you can see how the heads moved. Indeed, for any Object in a Museum, there should be sufficient information provided to show the visitor, at least roughly, how it worked. These Objects may have worked once, but restoration is impractical, or impossibly expensive; but still they have a story to tell. 


\subsection{Once-in-a-Blue-Moon Objects}

These are usually made of shining brass and are kept in glass cabinets where they cannot be sullied by dirty fingers. But this hardly helps the public to appreciate their significance. So, once every 50 years at least, they should be taken out and turned over by hand to show how they did whatever they were intended to do. And then you make a video of the operation, which can be seen on demand by the visiting Public and even sold for profit in the Museum shop. There are lots of Objects this could be done to, but I have my eye focussed on two of them in the Science Museum:

1. The piece of Babbage's Difference Engine. "Sacrilege" I can hear the Curators saying. But I know this works, because I have done it before with the smaller piece of that machine kept in the Computer Laboratory at Cambridge; it worked just fine (no adjustments needed) and we used it to produce a small table of squares and cubes.

2. Kelvin's Harmonic Analyser. By peering closely at it, I was able to work out what it did (which is not what I had expected it to do - Kelvin had a later machine for that). But it would be fascinating to watch it (or a video of it) in action, and it would be a brilliant tool for explaining what Fourier analysis is all about.

\subsection{Replicas}

Where the original machine no longer exists, but where its historical significance is sufficiently great, building a replica can be justified, though I am far from convinced this is so for machines which never existed physically (unless there is serious doubt as to whether they would actually have worked). The great advantage of a replica is that it is not an original to be preserved at all costs, and so you can experiment with it; moreover it still fulfils the objective of preserving the skills needed for its construction and use.

\subsection{Restoring Original Machines}

Museum curators can get somewhat paranoid about the destruction of valuable historical evidence, which is an inevitable accompaniment to restoration. For Hartree's differential analyser, we are keeping careful records of what we have done, and any part that is re-manufactured or otherwise not part of the original is painted blue. But where the Museum is prepared to allow it (and the simplest way to ensure that is for the donor to make it a condition) it has great advantages; for sure the skills are preserved, and the Public can see it in operation. But a word of warning here; the Public needs to see something worthwhile, to learn something new, to be impressed; a black box that simply dishes out correct results will not do. Ideally, the machine needs to be seen solving real (but perhaps small) problems of the type it was intended for. And don't forget to video the presentation for when demonstrations are not actually in progress. 
And another word of warning: make sure that plans are in place to ensure demonstrations will continue for whatever is the expected lifetime of the restoration. Museums can be exceedingly fickle; priorities and visions of their "Grand Objective" can change as fast as new Directors get appointed. So each project must have a "Champion", and a fairly ruthless one at that. Fortunately, the CSS is well placed to provide and support such Champions, and to ensure their succession as time takes its toll.

\subsection{Emulation}

Is this not an alternative to restoration? To some extent Yes; it preserves some (but not all) of the skills, it is much cheaper, and people can download the emulation and play with it at home. But it is best regarded as an adjunct to rather than a substitute for the real thing.

\section{Mindsets}

These change over time - even within the period from c.1950 to c.1980 upon which we are concentrating. They concern the issues which the computer designers of that time had to contend with, the choices they had to make, the technologies available to them, the methodologies that they used. We need to give the Public some feel for them, because they see present day computers as communication and image manipulation/display gadgets with jazzy interfaces for which our issues do not seem immediately relevant. Evidently, within our time-frame, computing was much more related to Mathematics, and giving the Public some feel for Mathematics is, in this age, an important objective in itself.

So what are the tools available to us? Good written material (and handouts) associated with the Objects, demonstrations of working exhibits, videos (which can go beyond simple images of the demonstrations - animation can be used to explain the underlying principles), hands-on contact with small actual objects; but, above all, interactive gadgets to illustrate the principles concerned (though they have to be of robust construction so as to be small-child-proof). So here are some examples, and some suggestions as to how to put these ideas across.

\subsection{Analog vs. Digital}

Analog computing has been going in and out of fashion for many centuries, and I don't think we have seen the last of it yet. Slide rules are the best tool to get the basic idea across; they were the pocket calculators of their day, every Museum will have several examples on show, and I have built a large one at MOSI for people to play with (and it aims to show how they were actually used, rather than simply demonstrating that it can multiply 2 by 3 to get 6 ). Another device that should be on hand (or on video) is a planimeter. 
To illustrate digital, you cannot beat a simple abacus (preferably a Russian shoty with ten beads per digit, although the ones in your glass cabinet are more likely to be Chinese or Japanese). It is an excellent tool to illustrate carry propagation (now there is an issue that caused much worry for the early computer designers); and you can then move up to a Brunsviga which again illustrates carry propagation, and then you go on to use it for multiplication, explaining that the same basic process must be occurring in every pocket calculator, computer and mobile telephone. Actually, the best way to show carry propagation in action is that video of Babbage's Difference Engine that I called for earlier, and if you could manage to get the hand cranked piece of his analytical engine working, that would illustrate anticipatory carry too.

\subsection{Decimal vs. Binary}

That certainly needs to be explained. MOSI has a nice interactive 4-bit binary adder/subtracter that gets well used.

\subsection{Serial vs. Parallel}

Nobody in his right mind would make a serial computer these days, but if they had never been made and seen to work at the start, nobody would have attempted to make a parallel one. The distinction is important if you want to illustrate delay-line storage.

\subsection{Logic Diagrams}

A topic not part of the usual Museum fare, but it is essential to tell how everything inside a computer is built out of a small number of basic logic elements - and, or and not. What is needed is an interactive mechanical model of an adder, clearly showing the individual gates and their interconnections. Make it however you will; steel balls rolling down grooves; mechanical representations of diodes (levers A and B pushing lever $\mathrm{C}$ down with a spring as a pull-up resistor makes an OR-gate - turn it upside down for an AND-gate). Let the kiddies set up two binary numbers, and then watch the carry propagating.

\subsection{Packaging}

Early computers were wired up just like wireless sets. Everything hard soldered in place, so if it broke, it took you hours to locate the fault, mend it, and get it running again. So they invented removable packages with simple gates on them. So examples of such packages should be on display (and that is why I suggested leaving the computer door open so they can be seen). If you can arrange a simple binary counter made out of packages and a scope to show it counting up, so much the better.

Now, of course, components are much more reliable, so everything is again hard soldered onto a motherboard (and if that breaks, you just throw it away and get another one - that is called "progress"). 


\subsection{Order Codes}

A lot of effort was made in the early days to get these right. Writing in machine code is unfashionable these days, but people need to realise that it is still there behind the scenes, whatever programming language is used. If you are running an emulator for something with a nice simple order code (e.g. the Baby or a Pegasus, ignoring all the drum stuff), then you should provide an opportunity for people to write their own small programs. Nothing complicated: "Squares and Cubes" or "Fibonacci Numbers" are quite sufficient to get the basic idea across.

\subsection{Input/Output}

Nobody these days has ever seen punched paper tape, let alone a teleprinter. So it needs to be on view. At MOSI, even though our Pegasus is not working, it is planned to commission the paper tape equipment and attach it to the emulator. This will make demonstrations much more interesting, because you can see things happening, which is an essential feature of any demonstration.

The same goes for punched cards, with the addition that they were in use long before computers came on the scene. Showing a full tabulator in operation is probably too much (leave that for the videos), but if you can lay your hands on a working card sorter, then that can make a most spectacular demonstration; moreover, it is easy to explain how it works, and it is fairly easy to maintain.

\subsection{Memory}

There is a progression - Williams tubes, through delay lines, core stores, and up to ICs - and a corresponding exponential growth in capacity. There should be exhibits to illustrate this progression. An interesting interactive would be a single core plane, with pushbuttons to select a row and column for current to flow through, a knob to determine the direction of the current (write or read), and a CRT, or at least a LED, to show whether a 1 or a 0 had been stored there.

\subsection{External Storage}

Tape decks should be in evidence (though having a working one might be a step too far). A drum would be fine, so long as you can see inside it. Exchangeable discs so people can gawk at how few megabytes they held, compared to their huge bulk. Floppy discs, and their drives (opened up). And of course a sectioned hard drive as already mentioned.

\subsection{Software}

This is a huge topic, and I am not sure that a Museum is the right place to exhibit it. But it does need to be preserved, and that is a whole new ballpark which I do not propose to enter here. 


\section{Videos}

You will have noticed my frequent mention of these, and I am surprised that Museums do not already make greater use of them, and not just for computers. Every gallery should have one, with a well-structured menu so people can choose exactly what they would like to see. Clearly, they should illustrate the Objects on local display, but there is the opportunity to go further, showing Objects from other Museums, and exploring underlying concepts in more detail, perhaps with the aid of animations.

\section{And Finally}

There is a temptation for Museums to concentrate overmuch on the quality of their displays; but this can easily lead to a triumph of Form over Content. Museum Managers bear a responsibility to ensure their visitors come away knowing more than when they came in. At their best, they can provide brilliant solutions for bringing this about. But, sadly, sometimes they can also be a part of the problem.

Acknowledgements. Thanks are due to Peter Onion, who presented this paper to the Conference at excruciatingly short notice when I was unwell. 